ELSEVIER Applied Mathematics and Computation 173 (2006) 350-359

www.elsevier.com/locate/amc

\title{
A novel algorithm for uncertain portfolio selection
}

\section{Jih-Jeng Huang a, Gwo-Hshiung Tzeng b,c,*, Chorng-Shyong Ong ${ }^{\mathrm{a}}$}

${ }^{a}$ Department of Information Management, National Taiwan University, No. 1, Sec. 4, Roosevelt Road, Taipei 106, Taiwan

${ }^{\mathrm{b}}$ Institute of Management of Technology and Institute of Traffic and Transportation College of Management, National Chiao Tung University, 1001 Ta-Hsueh Road, Hsinchu 300, Taiwan

${ }^{\mathrm{c}}$ Department of Business Administration, Kainan University, No. 1, Kai-Nan Road, Luchu, Taoyuan 338, Taiwan

\begin{abstract}
In this paper, the conventional mean-variance method is revised to determine the optimal portfolio selection under the uncertain situation. The possibilistic area of the return rate is first derived using the possibisitic regression model. Then, the Mellin transformation is employed to obtain the mean and the risk by considering the uncertainty. Next, the revised mean-variance model is proposed to deal with the problem of uncertain portfolio selection. In addition, a numerical example is used to demonstrate the proposed method. On the basis of the numerical results, we can conclude that the proposed method can provide the more flexible and accurate results than the conventional method under the uncertain portfolio selection situation.

(C) 2005 Elsevier Inc. All rights reserved.
\end{abstract}

\footnotetext{
* Corresponding author.

E-mail address: u5460637@ms16.hinet.net (G.-H. Tzeng).
} 
Keywords: Mean-variance method; Portfolio selection; Possibilistic regression; Mellin transformation

\section{Introduction}

The mean-variance approach was proposed by Markowitz to deal with the portfolio selection problem [1]. A decision-maker can determine the optimal investing ratio to each security based on the sequent return rate. The formulation of the mean-variance method can be described as follows [1-3]:

$$
\begin{array}{ll}
\min & \sum_{i=1}^{n} \sum_{j=1}^{n} \sigma_{i j} x_{i} x_{j}, \\
\text { s.t. } & \sum_{i=1}^{n} \mu_{i} x_{i} \geqslant E, \\
& \sum_{i=1}^{n} x_{i}=1, \\
& x_{i} \geqslant 0 \quad \forall i=1, \ldots, n,
\end{array}
$$

where $\mu_{i}$ denotes the expected return rate of the $i$ th security, $\sigma_{i j}$ denotes the covariance coefficient between the $i$ th security and the $j$ th security, and $E$ denotes the acceptable least rate of the expected return.

It is clear that the accuracy of the mean-variance approach depends on the accurate value of the expected return rate and the covariance matrix. Several methods have been proposed to forecast the appropriate expected return rate and variance matrix such as the arithmetic mean method [1-3] and the regression-based method [4]. However, these methods only derive the precise expected return rate and covariance matrix and do not consider the problem of uncertainty. That is, since the decision-maker try to determine the optimal portfolio strategy to gain the maximum profits in the future, how can we ignore the future uncertainty. We should highlight that the possible area of the return rate and the covariance matrix should be derived for the decision-maker to determine the future optimal portfolio selection strategy. In addition, these methods are based on the large sample theory and cannot obtain a satisfactory solution in the small sample situation [5].

In this paper, the possible area of the return rate and the covariance matrix are derived using the asymmetrical possibilistic regression. Then, the Mellin transformation is employed to calculate the uncertain return rate and the variance with the specific distribution. Finally, the optimal portfolio selection model can be reformulated based on the concepts above. In addition, a numerical example is used to illustrate the proposed method and compared with the 
conventional mean-variance method. On the basis of the simulated results, we can conclude that the proposed method can provide the better portfolio selection strategy than the conventional mean-variance method by considering the situation of uncertainty.

The remainder of this paper is organized as follows. The possibilistic regression model is discussed in Section 2. The Mellin transformation and the proposed method are presented in Section 3. A numerical example, which is used to illustrate the proposed method and compare with the mean-variance method, is in Section 4. The discussions of the numerical results are presented in Section 5 and the conclusions are presented in the last section.

\section{Possibilistic regression}

The possibilistic regression model was first proposed by Tanaka and Guo [6] to reflect the fuzzy relationship between the dependent and the independent variables. The upper and the lower regression boundaries are used in the possibilistic regression to reflect the possibilistic distribution of the output values. By solving the linear programming (LP) problem, the coefficients of the possibilistic regression can easy be obtained.

Next, we describe the possibilistic regression model [6] to obtain the uncertain return rate and the variance as follows. In order to exactly obtain the results, we extend the symmetrical fuzzy numbers to the asymmetrical fuzzy numbers. The general form of a possibilistic regression can be expressed as

$$
\boldsymbol{y}=A_{0}+A_{1} x_{1}+\cdots+A_{n} x_{n}=\boldsymbol{A}^{\prime} \boldsymbol{x},
$$

where $A_{i}$ is a asymmetrical possibilistic regression coefficient which is denoted as $\left(a_{i}-c_{i L}, a_{i}, a_{i}+c_{i R}\right)$. In order to obtain the minimum degree of uncertainty, the fitness function of the possibilistic regression can be defined as

$$
\min _{\boldsymbol{a}, \boldsymbol{c}} J=\sum_{j=1, \ldots, m} \boldsymbol{c}_{L}^{\prime}\left|x_{j}\right|+\boldsymbol{c}_{R}^{\prime}\left|x_{j}\right| .
$$

In addition, the dependent variable should be restricted to satisfy the following two equations:

$$
\begin{gathered}
y_{j} \geqslant \boldsymbol{a}^{\prime} \boldsymbol{x}_{j}-\boldsymbol{c}_{L}^{\prime}\left|x_{j}\right|, \\
y_{j} \leqslant \boldsymbol{a}^{\prime} \boldsymbol{x}_{j}+\boldsymbol{c}_{R}^{\prime}\left|x_{j}\right| .
\end{gathered}
$$

On the basis of the concepts above, we can obtain the formulation of a possibilistic regression model as follows: 


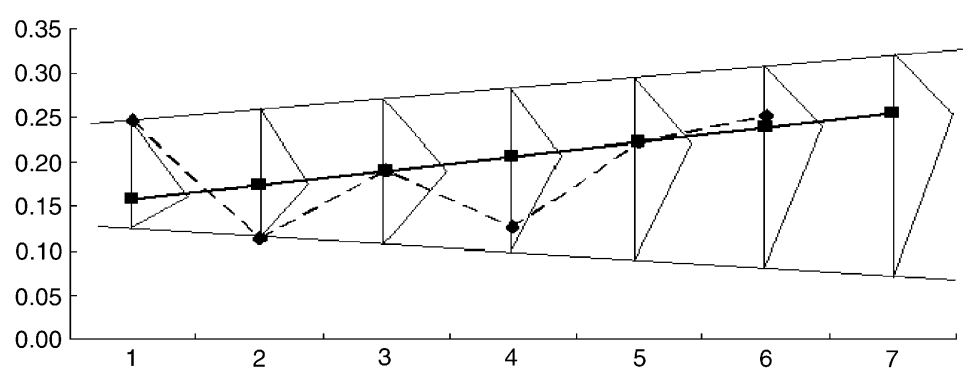

Fig. 1. The possibilistic area of the return rate and the variance.

$$
\begin{array}{ll}
\min _{\boldsymbol{a}, \boldsymbol{c}} & J=\sum_{j=1, \ldots, m} \boldsymbol{c}_{L}^{\prime}\left|\boldsymbol{x}_{j}\right|+\boldsymbol{c}_{R}^{\prime}\left|\boldsymbol{x}_{j}\right|, \\
\text { s.t. } & y_{j} \geqslant \boldsymbol{a}^{\prime} \boldsymbol{x}_{j}-\boldsymbol{c}_{L}^{\prime}\left|\boldsymbol{x}_{j}\right|, \\
& y_{j} \leqslant \boldsymbol{a}^{\prime} \boldsymbol{x}_{j}+\boldsymbol{c}_{R}^{\prime}\left|\boldsymbol{x}_{j}\right|, \quad j=1, \ldots, m, \\
& \boldsymbol{c}_{L}, \boldsymbol{c}_{R} \geqslant 0 .
\end{array}
$$

By solving the mathematical programming model above, we can obtain the uncertain return rate and the variance of the security with the specific distribution in the future.

Next, we depict a graph, as shown in Fig. 1, to describe the concept of the proposed method. Suppose we have six period return rates of stocks and we want to determine the optimal investing rate to each stock in period 7 . Let the broken line denotes the trend of the return rate of a stock. Then, we can obtain the upper, the lower, and the center possibilistic regressions using Eq. (6) to derive the possibilistic area of the return rate of period 7. Note that the triangular possibilistic distribution is used in this example. However, other possibilistic distributions can be employed using the same concepts.

We should highlight that the triangular area in period 7 because it denotes the distribution of the possible return rate and variance of the stock. That is, the decision-maker should incorporate the information above to determine the optimal investing rate to each stock. However, since the possibilistic area may be triangular, uniform, or other distributions, the problem is how to efficiently and effectively calculate the possible return rate and the variance. Next, the Mellin transformation is described to overcome this problem.

\section{The Mellin transformation}

Given a random variable, $x \in R^{+}$, the Mellin transformation, $M(s)$, of a probability density function (pdf) $(f(x))$ can be defined as 
Table 1

The properties of the Mellin transformation

\begin{tabular}{lll}
\hline Properties of Mellin transformation & $Y=h(x)$ & $M(s)$ \\
\hline Scaling property & $a x$ & $a^{-s} M(s)$ \\
Multiplication by $x^{a}$ & $x^{a} f(x)$ & $M(s+a)$ \\
Rising to a real power & $f\left(x^{a}\right)$ & $a^{-1} M\left(\frac{s}{a}\right),(a>0)$ \\
Inverse & $x^{-1} f\left(x^{-1}\right)$ & $M(1-s)$ \\
Multiplication by $\ln x$ & $\ln x f(x)$ & $\frac{\mathrm{d}}{\mathrm{d} s} M(s)$ \\
Derivative & $\frac{\mathrm{d}^{k}}{\mathrm{~d} s^{k}} f(x)$ & $\frac{\Gamma(s)}{\Gamma(s-k)}$ \\
\hline
\end{tabular}

$$
M\{f(x) ; s\}=M(s)=\int_{0}^{\infty} f(x) x^{s-1} \mathrm{~d} x .
$$

Let $h$ is a measurable function on $\mathbb{R}$ into $\mathbb{R}$ and $Y=h(x)$ is a transformed random variable. Then, some properties of the Mellin transformation can be described as shown in Table 1. For example, if $Y=a x$ then the scaling property can be expressed as

$$
M\{f(a x) ; s\}=\int_{0}^{\infty} f(a x) x^{s-1} \mathrm{~d} x=a^{-s} \int_{0}^{\infty} f(a x)(a x)^{s-1} \mathrm{~d} x=a^{-s} M(s) .
$$

Next, let a continuous non-negative random variable, $X$, the $n$th moment of $X$ can be defined as

$$
E\left(X^{n}\right)=\int_{0}^{\infty} x^{n} f(x) \mathrm{d} x
$$

Then, by setting $n=1$, the mean of $X$ can be expressed as

$$
E(X)=\int_{0}^{\infty} x f(x) \mathrm{d} x
$$

and the variance of $X$ can be calculated by

$$
\sigma_{x}^{2}=E\left(X^{2}\right)-[E(X)]^{2} .
$$

Since the relationship between the $n$th moment and the Mellin transformation of $X$ can be linked using the equation

$$
E\left(X^{n}\right)=\int_{0}^{\infty} x^{(n+1)-1} f(x) \mathrm{d} x=M\{f(x) ; n+1\},
$$

the mean and the variance of $X$ can be calculated by

$$
\begin{aligned}
& E(X)=M\{f(x) ; 2\}, \\
& \sigma_{x}^{2}=M\{f(x) ; 3\}-\{M\{f(x) ; 2\}\}^{2} .
\end{aligned}
$$

From Eqs. (12) and (13), we can efficiently calculate the mean and the variance of any distribution using the Mellin transformation. In practice, the uniform, the triangular and the trapezoidal distributions are usually used and their 
Table 2

The Mellin transformation of three probability density functions

\begin{tabular}{lll}
\hline Distribution & Parameters & $M(s)$ \\
\hline Uniform & $\mathrm{UNI}(a, b)$ & $\frac{b^{s}-a^{s}}{s(b-a)}$ \\
Triangular & $\operatorname{TRI}(l, m, u)$ & $\frac{2}{(u-l) s(s+1)}\left[\frac{u\left(u^{s}-m^{s}\right)}{(u-m)}-\frac{l\left(m^{s}-l^{s}\right)}{(m-l)}\right]$ \\
Trapezoidal & $\operatorname{TRA}(a, b, c, d)$ & $\frac{2}{(c+d-b-a) s(s+1)}\left[\frac{\left(d^{s+1}-c^{s+1}\right)}{(d-c)}-\frac{\left.b^{s+1}-a^{s+1}\right)}{(b-a)}\right]$ \\
\hline
\end{tabular}

corresponding Mellin transformations can be summarized as shown in Table 2. More Mellin transformation for other probability density functions can refer to [7].

On the basis of Table 2, we can efficiently derived the values of the mean and the variance respect to the specific distribution by calculating $M(2)$ and $M(3)$. Next, we can reformulate the conventional mean-variance method as shown in the following mathematical programming model to consider the impact of uncertainty:

$$
\begin{array}{ll}
\min & \sum_{i=1}^{n} x_{i} x_{i} \cdot\left[M_{i}(3)-M_{i}(2)^{2}\right]+\sum_{i=1}^{n} \sum_{j=1}^{n} x_{i} x_{j} \sigma_{i j}, \\
\text { s.t. } & \sum_{i=1}^{n} x_{i} M_{i}(2) \geqslant E, \\
& \sum_{i=1}^{n} x_{i}=1, \\
& x_{i} \geqslant 0 \forall i=1, \ldots, n,
\end{array}
$$

where the first part of the objective function denotes the next period risk of the security, the second part of the objective function denotes the unsystematic risk which is considered in the mean-variance model. Next, we use a numerical example to illustrate the proposed method and to compare with the conventional method.

\section{A numerical example}

For simplicity, the possibilistic area of the return rate is represented as the triangular form in this numerical example. Suppose the historical sequent return rates of the five securities from periods $t-6$ to $t-1$ can be represented as shown in Table 3. The corresponding time chart of the five securities can also be depicted as shown in Fig. 2. Our concern here is to obtain the optimal portfolio selection strategy in the next period $t$.

First, we use the conventional mean-variance model to obtain the optimal portfolio selection strategy. To do this, the arithmetic mean and the covariance matrix can be calculated as shown in Tables 4 and 5 . 
Table 3

The historical return rates of the five securities

\begin{tabular}{lllllll}
\hline & \multicolumn{6}{l}{ Return rate } \\
\cline { 2 - 7 } & $t-6$ & $t-5$ & $t-4$ & $t-3$ & $t-2$ & $t-1$ \\
\hline Security 1 & 0.1686 & 0.1117 & 0.1149 & 0.1293 & 0.1397 & 0.1406 \\
Security 2 & 0.1330 & 0.1466 & 0.1741 & 0.1131 & 0.1022 & 0.1552 \\
Security 3 & 0.1698 & 0.1528 & 0.1302 & 0.1471 & 0.1139 & 0.1177 \\
Security 4 & 0.1750 & 0.1026 & 0.1543 & 0.1475 & 0.1158 & 0.1148 \\
Security 5 & 0.1291 & 0.1192 & 0.1491 & 0.1318 & 0.1377 & 0.1450 \\
\hline
\end{tabular}

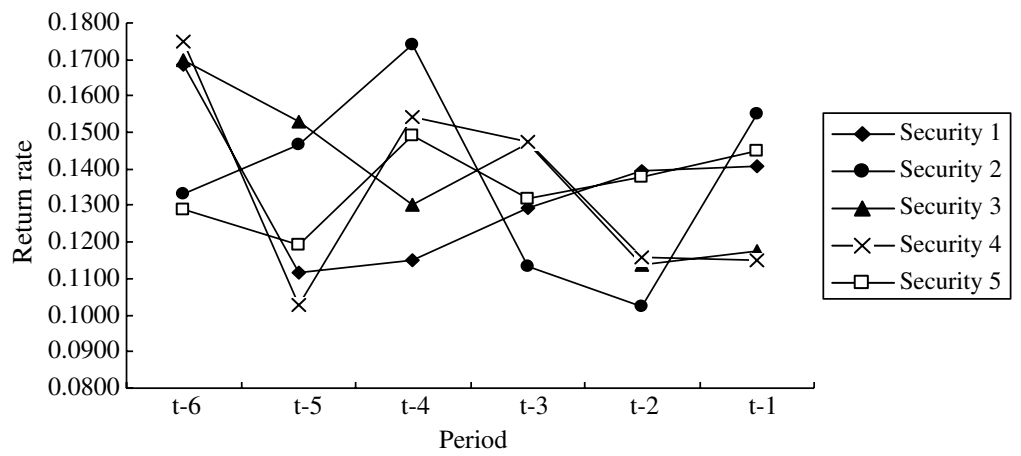

Fig. 2. The time chart of the five securities.

Table 4

Arithmetic mean of the expected return

\begin{tabular}{lllllll}
\hline Security & 1 & 2 & 3 & 4 & 5 & Average \\
\hline Arithmetic mean & 0.1341 & 0.1374 & 0.1386 & 0.1350 & 0.1353 & 0.136 \\
\hline
\end{tabular}

Table 5

The covariance matrix

\begin{tabular}{lrrrrr}
\hline & Security 1 & Security 2 & Security 3 & Security 4 & Security 5 \\
\hline Security 1 & 0.00036 & & & & \\
Security 2 & -0.00017 & 0.00060 & & & \\
Security 3 & 0.00010 & 0.00000 & 0.00039 & & \\
Security 4 & 0.00024 & 0.00004 & 0.00027 & 0.00066 & \\
Security 5 & 0.00000 & 0.00009 & -0.00014 & 0.00004 & 0.00010 \\
\hline
\end{tabular}

Let the acceptable least rate of the expected return is equal to its average return rate, we can obtain the optimal portfolio selection using Eq. (1) as shown in Table 8. 
Table 6

The possibilistic area, the mean and the variance

\begin{tabular}{llllll}
\hline & Security 1 & Security 2 & Security 3 & Security 4 & Security 5 \\
\hline Possibilistic & $(0.1117,0.1117$, & $(0.0868,0.1407$, & $(0.0890,0.0890$, & $(0.0646,0.0646$, & $(0.1500,0.1500$, \\
area & $0.1578)$ & $0.1741)$ & $0.1241)$ & $0.1294)$ & $0.1737)$ \\
Mean & 0.1306 & 0.1339 & 0.1007 & 0.0862 & 0.1579 \\
Variance & 0.000118 & 0.000323 & 0.000068 & 0.000233 & 0.000031 \\
\hline
\end{tabular}

Table 7

The new covariance matrix

\begin{tabular}{lrlrrl}
\hline & Security 1 & Security 2 & Security 3 & Security 4 & Security 5 \\
\hline Security 1 & 0.00031 & & & & \\
Security 2 & -0.00015 & 0.00052 & & & \\
Security 3 & 0.00010 & 0.00001 & 0.00051 & & \\
Security 4 & 0.00023 & 0.00006 & 0.00046 & 0.00086 & \\
Security 5 & -0.00001 & 0.00007 & -0.00022 & -0.00010 & 0.00015 \\
\hline
\end{tabular}

Table 8

The comparisons of the portfolio selections

\begin{tabular}{llllllll}
\hline Portfolio strategy & 1 & 2 & 3 & 4 & 5 & Return rate & Portfolio risk \\
\hline Conventional & 0.000 & 0.069 & 0.195 & 0.303 & 0.433 & 0.136 & 0.000056 \\
Proposed method & 0.136 & 0.070 & 0.141 & 0.118 & 0.535 & 0.136 & 0.000073 \\
\hline
\end{tabular}

Next, we use the proposed method to obtain the optimal portfolio selection as follows. In order to obtain the possibilistic area of the five securities, the possibilistic regression is employed. Then, using the Mellin transformation we can obtain the forecasting mean and risk of the securities by considering the situation of uncertainty as shown in Table 6 .

Furthermore, we incorporate the information of the forecasting mean to derive the second part of the objective function in Eq. (14), i.e. the covariance matrix, as shown in Table 7.

Finally, with the same acceptable least rate of the expected return rate we can obtain the optimal portfolio selection under the uncertain situation using Eq. (14). The comparison of the conventional and the proposed method can be described as shown in Table 8 .

From Table 8, it can be seen that the main difference is the portfolio selection in Securities 1 and 4. In the next section, we will discuss the irrational reason using the conventional method in our numerical example. 


\section{Discussions}

The mean-variance method is widely used in the finance area to deal with the portfolio selection problem. However, the conventional method does not consider the situation of future uncertainty and usually fails under the small sample situation. We can describe the shortcomings of the conventional method from its purpose and theory, respectively, as follows.

The purpose of the mean-variance approach is to determine the $t$ period optimal investing rate to each security based on the historical sequent return rate. The key is to forecast the $t$ period return rate as accurately as possible. However, it is clear that the arithmetic mean can only reflect the average states of the past return rate instead of the future. Although many regression-based methods have been proposed to overcome the problem, these methods must obey the assumption of the large sample theory and cannot be used in the small sample situation theoretically.

In addition, these methods cannot reflect the degree of uncertainty. Since we want to determine the optimal portfolio selection in the future, the information of future uncertainty should not be ignore in the model. In this paper, the possibilistic regression model is employed to derive the possible mean and the variance in the future. Then, the Mellin transformation is used to obtain the mean and the risk in the future by considering the uncertain situation. Finally, we can use the information above to reformulate the mean-variance method to obtain the optimal uncertain portfolio selection.

In order to highlight the shortcoming of the conventional method and to compare it with the proposed method, a numerical example is used in Section 4. Now, we can depict the time chart of Securities 1 and 4 to describe the irrational results using the conventional method as shown in Fig. 3.

From the time chart, it can be seen from Security 1 that there is an increase in the period of $t-4$ to $t-1$. It is rational to suppose Security 1 also has the

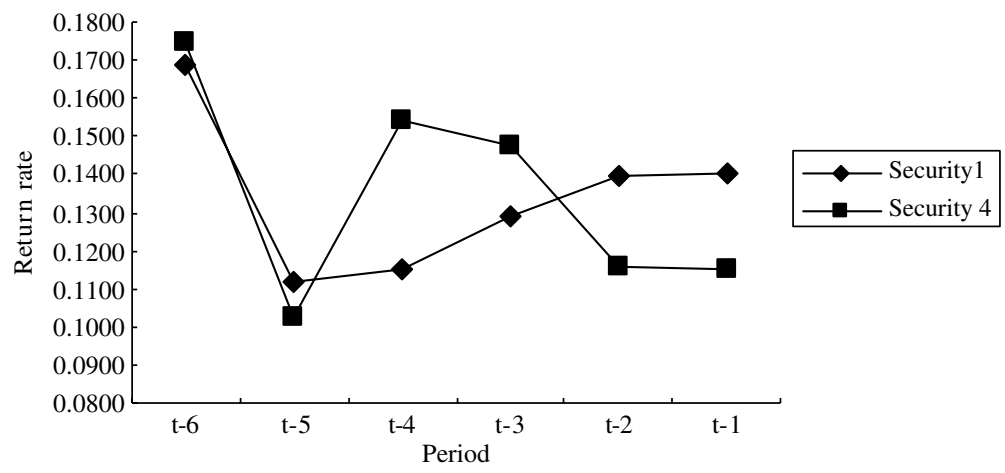

Fig. 3. The time chart of Securities 1 and 4. 
positive return rate in the period $t$. On the other hand, Security 4 shows the decrease since the period $t-4$, the optimal portfolio selection should eliminate the investing ratio in Security 4. On the basis of the numerical results, we can conclude that it is irrational to determine the uncertain portfolio selection using the conventional method. On the other hand, the proposed method can accurately reflect the deduction above. In addition, the proposed method can provide the more flexible portfolio alternatives. That is, a decision-maker can determine the optimal possibilistic distribution based on his domain knowledge or the empirical results to obtain the exactly portfolio selection strategy.

\section{Conclusions}

Portfolio selection problem has been a popular issue in the finance area since 1950s. However, the conventional mean-variance method can not provide the satisfactory solution under the uncertain portfolio selection and the small sample situations. In this paper, the possibilistic regression model is employed to derive the possibilistic area of the future return rate. The Mellin transformation, then, is used to obtain the mean and the risk by considering the uncertainty. Using the information above, we propose the revised mean-variance model which incorporates the degree of uncertainty to deal with the problem of portfolio selection. A numerical example is used to demonstrate the proposed method. On the basis of the numerical results, we can conclude that the proposed method can provide the more flexible and accurate results than the conventional method under the uncertain portfolio selection situation.

\section{References}

[1] H. Markowitz, Portfolio selection, J. Finance 7 (1) (1952) 77-91.

[2] H. Markowitz, Portfolio Selection: Efficient Diversification of Investments, Wiley, New York, 1959.

[3] H. Markowitz, Mean-Variance Analysis in Portfolio Choice and Capital Market, Basil Blackwell, New York, 1987.

[4] E.J. Elton, M.J. Gruber, Modern Portfolio Theory and Investment Analysis, Wiley, New York, 1995.

[5] E.J. Elton, M.J. Gruber, T.J. Urich, Are betas best? J. Finance 33 (5) (1978) 1357-1384.

[6] H. Tanaka, P. Guo, Possibilistic Data Analysis for Operations Research, Physica-Verlag, New York, 2001.

[7] K.P. Yoon, A probabilistic approach to rank complex fuzzy numbers, Fuzzy Sets Syst. 80 (2) (1996) 167-176. 